\title{
L'OMOGENITORIALITÀ TRA ASPIRAZIONE E DIRITTO - NOTE A MARGINE DELLA SENTENZA N. 230/2020 DELLA CORTE COSTITUZIONALE
}

\author{
HOMOGENITORIALITY BETWEEN ASPIRATION AND LAW \\ - REMARKS ON JUDGEMENT N. 230/2020 OF THE ITALIAN \\ CONSTITUTIONAL COURT
}

\author{
ILARIA AQUIRONI \\ Assegnista di ricerca in Diritto internazionale \\ Università degli Studi di Ferrara
}

Recibido: 16.06.2021 / Aceptado: 07.07.2021

DOI: https://doi.org/10.20318/cdt.2021.6249

\begin{abstract}
Riassunto: Una recente sentenza della Corte costituzionale offre lo spunto per riflettere sull'evoluzione giurisprudenziale italiana in materia di omogenitorialità, nonché per mettere in luce gli insoddisfacenti esiti cui la giurisprudenza perviene in assenza di una disciplina normativa organica in materia. Le soluzioni giurisprudenziali sin qui adottate fanno sì che il grado di tutela offerto dall'ordinamento italiano nei confronti del minore nato attraverso il ricorso alle tecniche procreative medicalmente assistite vari a seconda di fattori del tutto secondari rispetto all'obiettivo, primario, di tutela del suo superiore interesse. Non si discosta da tale rilievo la sentenza n. 230 del 2020, criticabile per la cautela mostrata dalla Corte costituzionale; per il vulnus alla tutela del superiore interesse del minore legato alla variabilità della garanzia offerta a seconda del luogo di nascita e dalla tecnica procreativa impiegata; per la portata differenziata del limite dell'ordine pubblico che di fatto corrobora; nonché per il perdurante impiego dell'insoddisfacente istituto dell'adozione in casi particolari quale strumento teso ad instaurare un legame tra genitore intenzionale e figlio minore.
\end{abstract}

Parole chiave: fecondazione eterologa, Corte costituzionale, omogenitorialità, riconoscimento degli status, formazione dell'atto di nascita,filiazione, maternità surrogata, adozione in casi particolari.

\footnotetext{
Abstract: A recent judgement of the Italian Constitutional Court serves as a basis to rethink on the evolution of the Italian jurisprudence concerning homogenitoriality, as well as to highlight the unsatisfactory results achieved, absent any omni comprehensive legislation on the issue. The judicial decisions adopted are such that the degree of protection accorded by the Italian legal system to the child born through the use of assisted reproductive technology depends on rather secondary factors compared to the primary goal of ensuring the best interests of the child. In this sense, Judgement no. 230 of 2020 of the Italian Constitutional Court is no different: critical remarks can be raised due to the cautious approach adopted by the Court; the vulnus to the protection of the best interests of the child, linked to the dependence of the degree of protection accorded to the child on the place of his or her birth, and on the assisted reproductive technique used; the variable extent of the public policy exception; and the still prevailing use of the "adoption in particular cases" as a (unsatisfactory) means to establish a legal relationship between the intended parent and the child.

Keywords: heterologous fertilization, Italian Constitutional Court, homogenitoriality, status recognition, issuance of the birth certificate, legal parent-child relation, surrogate motherhood, adoption in particular cases.
} 
Sommario: I. Rilievi introduttivi - II. La fattispecie oggetto dell'ordinanza di rimessione del Tribunale di Venezia - III. L'evoluzione della giurisprudenza della Corte di cassazione e della Corte costituzionale in materia di omogenitorialità - IV. L'orientamento della Corte costituzionale nella sentenza n. 230 del 2020 - V. Considerazioni critiche - 1. L'eccessiva cautela mostrata dalla Corte costituzionale -2 . Il vulnus alla tutela del superiore interesse del minore -3 . La portata differenziata del limite dell'ordine pubblico - 4. I limiti dell'istituto dell'adozione in casi particolari - VI. Conclusioni.

\section{Rilievi introduttivi}

1. Nell'epoca contemporanea si assiste ad una trasformazione del concetto di genitorialità, nel senso di una ridefinizione dei suoi confini ${ }^{1}$ : alla filiazione biologica, difatti, si affianca sempre più di frequente un'idea di genitorialità c.d. "sociale" o "di intenzione".

2. Le ragioni sottese a questo fenomeno sono molteplici, ancorché latamente riconducibili entro due osservazioni.

Per un verso, il progresso tecnico-scientifico ha interessato anche l'area medica e, nel quadro di quest'ultima, l'ambito della procreazione medicalmente assistita. Tale evoluzione ha consentito ad un numero crescente di single o di coppie - tanto omosessuali quanto eterosessuali - di fare ricorso a tecniche procreative che, attraverso il coinvolgimento di soggetti estranei alla coppia (siano essi donatori di gameti o madri surrogate), tendono al superamento dei limiti fisiologici o patologici connessi alla filiazione esclusivamente biologica².

Per altro verso, la società civile appare sempre più sensibile ed attenta verso le istanze di riconoscimento giuridico, e dunque di tutela, promosse dalla comunità LGBT. Nel quadro di tali istanze, si inscrivono anche quelle tese al soddisfacimento di un progetto familiare e del desiderio di genitorialità che prescinda dal requisito esclusivamente biologico della diversità di sesso tra i genitori.

3. A fronte di tali situazioni, i diversi ordinamenti giuridici nazionali hanno dato vita ad una pluralità di discipline, più o meno organiche, tese tanto a regolamentare la legittimità delle tecniche riproduttive in parola, quanto ad assicurare il riconoscimento dei legami che ne derivano ${ }^{3}$.

${ }^{1} \mathrm{Si}$ veda, in questo senso, O. FERACI, "Ai confini della genitorialità ovvero sulla maternità surrogata commerciale in assenza di legami genetici tra genitori intenzionali e figli", in A. SACCUCCI (a cura di), Paulo Pinto de Albuquerque, I diritti umani in una prospettiva europea, Opinioni dissenzienti e concorrenti (2016-2020), Napoli, Editoriale Scientifica, 2021, pp. 271-280, p. 271; ID., "Maternità surrogata conclusa all'estero e Convenzione europea dei diritti dell'uomo: Riflessioni a margine della sentenza Paradiso e Campanelli c. Italia", Cuadernos de Derecho Transnacional, n. 2, 2015, pp. 420-439, p. 421.

${ }^{2}$ Si parla, in particolare, per le coppie di sesso femminile, di fecondazione eterologa allorché nell'utero di una delle due donne componenti la coppia venga impiantato un ovocita dell'altra donna o di una donatrice anonima, fecondato con il seme di un donatore anonimo. Anche le coppie eterosessuali fanno sovente ricorso alla fecondazione eterologa: in questo senso, l'ovocita di una donatrice viene impiantato nell'utero della partner della coppia, dopo essere stato fecondato con il seme del coniuge o di un donatore. Le coppie di sesso maschile, per contro, ricorrono alla surrogazione di maternità, tecnica invero capace di suscitare importanti interrogativi bioetici e sociali, ancor prima che giuridici. In questa prospettiva, si parla di maternità surrogata tradizionale allorché la madre surrogata sia la madre genetica del nato; la tecnica sottende un contratto tra i genitori committenti e una donna fertile, la quale acconsente all'inseminazione artificiale con seme di uno dei committenti o di un donatore anonimo, e alla gestazione, impegnandosi a rinunciare, al momento del parto, alla responsabilità genitoriale sul nato. È definita maternità surrogata gestazionale quella forma di gestazione per altri, comunque regolata attraverso un contratto, in cui non vi sono legami genetici tra la madre surrogata e il bambino, essendo l'ovocita prelevato da una donatrice, fecondato in vitro - vuoi con seme vuoi di uno dei committenti, vuoi di donatore anonimo - e solo successivamente impiantato nell'utero della madre surrogata per la prosecuzione della gestazione. Gli accordi sottesi a ciascuna delle due forme di maternità surrogata possono essere commerciali o altruistici: nel primo caso, alla madre surrogata spetta un compenso economico mentre, nel secondo caso, la madre surrogata porta a termine la gravidanza a titolo essenzialmente gratuito, essendole esclusivamente dovuto un indennizzo per le spese sostenute durante la gravidanza e per il parto. Sulle implicazioni internazionalprivatistiche della procreazione medicalmente assistita, si veda C. CAmpiglio, Procreazione assistita e famiglia nel diritto internazionale, Padova, Cedam, 2003.

${ }^{3}$ Per una ricognizione dei diversi approcci adottati in seno agli Stati membri dell'Unione europea in relazione alla pratica della maternità surrogata, si veda Dg for Internal Policies - Policy Department - Citizens' Rights and Constitutional Affairs, $A$ Comparative Study on the Regime of Surrogacy in EU Member States, 2013, pp. 200-351, disponibile sul sito www.europarlamento.eu. Si veda inoltre, in generale, K. Trimmings, P. Beaumont, General Report on Surrogacy, in K. Trimmings, P. Beaumont (a cura di), International Surrogacy Arrangements: Legal Regulation at the International Level, Oxford, Hart Publishing, 2013, pp. 441 ss. 
4. L'ordinamento italiano, dal canto suo, rimane o ancorato a parametri normativi restrittivi o del tutto silente tanto rispetto alle nuove frontiere delle tecniche riproduttive assistite, quanto alle istanze di riconoscimento e tutela degli status familiari che ne discendono.

Da un lato, se la legge 40/2004 ${ }^{4}$ rimane tuttora l'unico tentativo di dar vita ad una disciplina normativa organica in materia di procreazione medicalmente assistita, occorre rammentare che le tecniche riproduttive assistite ivi contemplate sono fruibili esclusivamente da coppie di sesso diverso ${ }^{5}$. In altre parole, nell'ordinamento italiano, l'accesso alla procreazione medicalmente assistita rimane appannaggio esclusivo di quelle coppie, composte da individui di sesso diverso, che non riescano a soddisfare il proprio desiderio di genitorialità a causa di impedimenti di natura patologica, ovverosia derivanti da infertilità o sterilità. Ciò, essenzialmente, in ragione della funzione eminentemente terapeutica attribuita dal legislatore italiano alle tecniche procreative medicalmente assistite.

D'altra parte - e in risposta solo parziale alle istanze di riconoscimento e tutela promosse dalla comunità LGBT - solamente nel 2016 il legislatore italiano è pervenuto ad una regolamentazione delle unioni civili omosessuali'. La c.d. 'Legge Cirinnà', tuttavia, disciplina esclusivamente il rapporto tra i partner dell'unione civile e i diritti e doveri nascenti dalla stessa', senza però riconoscere il legame genitoriale tra la coppia e i figli eventualmente nati attraverso le tecniche procreative assistite, o nei confronti di eventuali figli del partner.

5. L'insufficiente sostrato normativo causa, da un lato, la proliferazione del c.d. turismo riproduttivo $^{8}$, ovverosia di quelle particolari forme di esercizio della mobilità transnazionale tese ad avere accesso a tecniche procreative altrimenti vietate o disciplinate in maniera più restrittiva nell'ordinamento di origine.

Dall'altro lato, ed in connessione con il profilo poc'anzi indicato, nel silenzio del legislatore si moltiplicano le pronunce giurisprudenziali di merito, di legittimità e costituzionali, tese a rispondere alle istanze di stabilimento o riconoscimento della filiazione avvenuta a seguito del ricorso alle diverse tecniche di procreazione medicalmente assistita. Nell'arco di poco più di un quinquennio, le sole Corti di legittimità e costituzionale hanno reso non meno di dieci pronunce tese, ciascuna, a fornire un tassello del complessivo mosaico in tema di omogenitorialità.

6. Una recente sentenza della Corte costituzionale, la n. 230 del $2020^{9}$, offre lo spunto per riflettere sull'evoluzione giurisprudenziale italiana in materia di stabilimento della filiazione omogenito-

\footnotetext{
${ }^{4}$ Legge n. 40/2004, del 19 febbraio, "Norme in materia di procreazione medicalmente assistita", in G.U. 24 febbraio 2004, n. 45. Si vedano, ex multis, R. VILLANI, La procreazione assistita: la nuova Legge 19 febbraio 2004, n. 40, Torino, Giappichelli, 2004; F. SANTOsuosso, La procreazione medicalmente assistita: commento alla Legge 19 febbraio 2004, n. 40, Milano, Giuffré, 2004.

${ }^{5}$ In particolare, l'Articolo 5 della legge 40/2004, nell'indicare i requisiti soggettivi per l'accesso alle tecniche procreative medicalmente assistite, precisa che possono accedervi "coppie di maggiorenni di sesso diverso, coniugate o conviventi, in età potenzialmente fertile, entrambi viventi". Occorre inoltre precisare che, in Italia, la pratica della maternità surrogata è vietata, oltre che penalmente sanzionata, dall'Articolo 12, comma 6, della legge 40/2004 alla luce del quale "(c)hiunque, in qualsiasi forma, realizza, organizza o pubblicizza la commercializzazione di gameti o di embrioni o la surrogazione di maternità è punito con la reclusione da tre mesi a due anni e con la multa da 600.000 a un milione di euro".

${ }^{6}$ Legge 76/2016, del 20 maggio, "Regolamentazione delle unioni civili tra persone dello stesso sesso e disciplina delle convivenze", in G.U. 21 maggio 2016, n. 118. Si veda, ex multis, E. DE GöTzEN, "Recognition of same-sex marriages, overcoming gender barriers in Italy and the Italian law no. 76/2016 on civil unions. First remarks", in Cuadernos de Derecho Transnacional, 2017, vol. 2, pp. 194-208.

${ }^{7} \mathrm{Si}$ veda, a titolo meramente esemplificativo, l'Articolo 1, comma 11 della legge n. 76/2016, ai sensi del quale "(c)on la costituzione dell'unione civile tra persone dello stesso sesso le parti acquistano gli stessi diritti e assumono i medesimi doveri; dall'unione civile deriva l'obbligo reciproco all'assistenza morale e materiale e alla coabitazione. Entrambe le parti sono tenute, ciascuna in relazione alle proprie sostanze e alla propria capacità di lavoro professionale e casalingo, a contribuire ai bisogni comuni".

${ }^{8}$ Sulle implicazioni internazionalprivatistiche del tema del turismo riproduttivo, si vedano D. SINDRES, "Le tourisme procréatif et le droit international privé", in Journal du droit international, 2015, pp. 429-504; H. Fulchiron, "La lutte contre le tourisme procréatif: vers un instrument de coopération internationale", in Journal du Droit International, 2012, pp. 563-588; J.-J. Lemouland, "Le tourisme procréatif”, in Actualité du droit international privé de la famille, 2000, pp. 24-33.

${ }^{9}$ Corte costituzionale, 20 ottobre 2020, depositata 4 novembre 2020, n. 230, in G.U. 11 novembre, n. 46, disponibile sul sito della Corte costituzionale www.cortecostituzionale.it.
} 
riale e sulle implicazioni che ne conseguono, nonché per mettere in luce gli insoddisfacenti esiti cui la giurisprudenza perviene, in mancanza di una disciplina normativa organica in materia di procreazione medicalmente assistita e riconoscimento degli status che ne derivano.

7. Tali soluzioni - vale la pena anticiparlo sin d'ora - fanno sì che il grado di tutela offerto dall'ordinamento italiano nei confronti del minore nato attraverso il ricorso alla procreazione medicalmente assistita vari a seconda di fattori del tutto secondari rispetto all'obiettivo, primario, di tutela del suo superiore interesse.

8. Dopo una breve ricostruzione della vicenda da cui trae origine la sentenza in commento, il presente scritto si propone di ripercorrere le principali pronunce giurisprudenziali rese dalla Corte di cassazione e dalla Corte costituzionale in materia di omogenitorialità. Infine, l'analisi della decisione n. 230 del 2020 resa dalla Corte consentirà di mettere in rilievo, per un verso e da un punto di vista formale, le criticità dell'approccio "a mosaico" sin qui adottato e, per altro verso e da un punto di vista sostanziale, le contraddizioni cui tali decisioni pervengono.

\section{La fattispecie oggetto dell'ordinanza di rimessione del Tribunale di Venezia}

9. La questione di legittimità costituzionale trae origine dalla vicenda di due donne italiane unite civilmente, recatesi all'estero al fine di ricorrere, l'una con il consenso dell'altra, alla fecondazione eterologa, pratica generalmente vietata in Italia ${ }^{10} \mathrm{e}$ in ogni caso preclusa alle coppie dello stesso sesso ${ }^{11}$. La nascita del figlio, avente un legame genetico solamente con una delle due donne, era avvenuta in Italia. A fronte del rifiuto opposto dall'Ufficiale di Stato civile di indicare nel certificato di nascita tanto la madre biologica quanto la madre intenzionale, e dell'indicazione da parte dello stesso della sola madre partoriente, le due donne avevano instaurato dinanzi al Tribunale di Venezia un procedimento di volontaria giurisdizione, chiedendo la rettificazione dell'atto di nascita ${ }^{12}$.

10. Il Tribunale di Venezia, non ritenendo di poter aderire all'orientamento giurisprudenziale che sostiene un'interpretazione evolutiva del quadro normativo esistente, aveva deciso di sollevare una questione di legittimità dinanzi alla Corte costituzionale.

Con l'ordinanza di rimessione n. 108 del $2019^{13}$, il Tribunale di Venezia aveva dunque sottoposto al vaglio della Corte costituzionale l'Articolo 1, comma 20 della legge 76/2016 ${ }^{14}$, nella parte in cui limita la tutela delle coppie di donne omosessuali unite civilmente ai soli diritti e doveri nascenti dall'unione

${ }^{10}$ La preclusione nei confronti della procreazione medicalmente assistita era incondizionata nella versione originaria dell'Articolo 4, comma 3, della legge 40/2004. La norma è stata tuttavia dichiarata costituzionalmente illegittima con la sentenza della Corte costituzionale del 9 aprile 2014, depositata il 10 giugno 2014, n. 162 in G.U. 18 giugno 2014, p 1 ss., nella parte in cui proibiva il ricorso a tale tecnica riproduttiva nel caso in cui fosse stata diagnosticata una patologia che sia causa di sterilità o infertilità assolute ed irreversibili. Sulla sentenza, si vedano, C. CAMPIGLIO, "Norme italiane sulla procreazione assistita e parametri costituzionali: il ruolo creativo della giurisprudenza", in Rivista di diritto internazionale privato e processuale, 2014, pp. 481-516; S. Tonolo, "Il diritto alla genitorialità nella sentenza della Corte costituzionale che cancella il divieto di fecondazione eterologa: profili irrisolti e possibili soluzioni”, in Rivista di diritto internazionale, 2014, pp. 1123-1147.

${ }^{11}$ Cfr., supra, nota 5.

${ }^{12}$ Il ricorso era stato promosso ai sensi dell'Articolo 95 del Decreto del Presidente della Repubblica n. 396/2000, del 3 novembre, "Regolamento per la revisione e la semplificazione dell'ordinamento dello stato civile, a norma dell'articolo 2, comma 12, della legge 15 maggio 1997, n. 127”, in. G.U. 30 dicembre 2000, n. 303.

${ }^{13}$ Tribunale di Venezia, sez. II civile, Ordinanza del 3 aprile 2019, in G.U. 10 luglio 2019, n. 28, pp. 60 ss.

${ }^{14}$ L'Articolo 1, comma 20, della 1. 76/2016 testualmente dispone: "Al solo fine di assicurare l'effettività della tutela dei diritti e il pieno adempimento degli obblighi derivanti dall'unione civile tra persone dello stesso sesso, le disposizioni che si riferiscono al matrimonio e le disposizioni contenenti le parole «coniuge», «coniugi» o termini equivalenti, ovunque ricorrono nelle leggi, negli atti aventi forza di legge, nei regolamenti nonché negli atti amministrativi e nei contratti collettivi, si applicano anche ad ognuna delle parti dell'unione civile tra persone dello stesso sesso. La disposizione di cui al periodo precedente non si applica alle norme del codice civile non richiamate espressamente nella presente legge, nonché alle disposizioni di cui alla legge 4 maggio 1983, n. 184. Resta fermo quanto previsto e consentito in materia di adozione dalle norme vigenti”. 
civile, e l'Articolo 29, comma 2, del D.P.R. 396/2000 ${ }^{15}$, nella parte in cui limita la possibilità di indicare il nome del solo genitore legittimo, nonché di quelli che rendono o hanno dato il consenso ad essere nominati, e non anche alle donne tra loro unite civilmente e siano ricorse all'estero alla procreazione medicalmente assistita.

11. Secondo il Tribunale di Venezia, il combinato disposto delle due norme in parola sarebbe in contrasto con l'Articolo 2 della Costituzione ${ }^{16}$, nella misura in cui, essendo le norme sulla procreazione medicalmente assistita non applicabili alle coppie di donne unite civilmente, non consentono, per queste ultime, la realizzazione del "diritto fondamentale alla genitorialità dell'individuo, sia come soggetto singolo che nelle formazioni sociali ove svolge la sua personalità" ${ }^{17}$.

12. Sussisterebbe inoltre un contrasto con l'Articolo 3, primo e secondo comma ${ }^{18}$, a causa della disparità di trattamento basata sull'orientamento sessuale e il reddito, nella misura in cui impedisce il riconoscimento del legame genitoriale tra il genitore d'intenzione e il figlio, allorché quest'ultimo sia nato in Italia, mentre consente la trascrizione di un atto di nascita di un minore nato all'estero, regolarmente ivi redatto e contenente l'indicazione di ambedue le madri. Le summenzionate norme darebbero luogo ad una discriminazione anche nei confronti del nato, atteso che, in osservanza al principio di uguaglianza, dovrebbe essere garantita a tutti i nati uguale tutela, di fatto prescindendo dalle caratteristiche - omosessuale o eterosessuale - della relazione tra i genitori ${ }^{19}$.

13. Le predette norme sarebbero inoltre in contrasto con l'Articolo $30^{20}$, sia in favore degli adulti che del nato, in quanto non rispetterebbero il principio di tutela della filiazione, per il quale, secondo il Tribunale di Venezia, sarebbe opportuna una concezione progressista della stessa, che imporrebbe di discostarsi da una dimensione meramente "naturalistico-fattuale", per lasciare spazio all'affermazione di un "diritto pretensivo", realizzabile attraverso le tecniche di procreazione medicalmente assistita e limitabile solo in vista di un bilanciamento con interessi aventi pari rango ${ }^{21}$.

14. Esisterebbe, secondo il giudice rimettente, anche un contrasto con l'Articolo 117 primo com$\mathrm{ma}^{22}$, in relazione all'Articolo 24, paragrafo 3 della Carta dei diritti fondamentali dell'Unione europea, agli Articoli 8 e 14 della Convenzione europea dei diritti dell'uomo, e all'Articolo 2 della Convenzione delle Nazioni Unite sui diritti dell'infanzia, che imporrebbero la tutela del diritto alla bigenitorialità ${ }^{23}$.

${ }^{15}$ Ai sensi dell'Articolo 29, comma 2, del D.P.R 396/2000, “(n)ell'atto di nascita sono indicati il luogo, l'anno, il mese, il giorno e l'ora della nascita, le generalità, la cittadinanza, la residenza dei genitori del figlio nato nel matrimonio nonché di quelli che rendono la dichiarazione di riconoscimento del figlio nato fuori del matrimonio e di quelli che hanno espresso con atto pubblico il proprio consenso ad essere nominati, il sesso del bambino e il nome che gli viene dato ai sensi dell'articolo 35".

${ }^{16}$ Ai sensi dell'Articolo 2 della Costituzione "(1)a Repubblica riconosce e garantisce i diritti inviolabili dell'uomo, sia come singolo, sia nelle formazioni sociali ove si svolge la sua personalità, e richiede l'adempimento dei doveri inderogabili di solidarietà politica, economica e sociale".

${ }^{17} \mathrm{Si}$ veda, sul punto, il § 3. II., lett. a) dell'Ordinanza di rimessione, spec. p. 65.

${ }^{18}$ L'Articolo 3 della Costituzione prevede che " 1 . Tutti i cittadini hanno pari dignità sociale e sono eguali davanti alla legge, senza distinzione di sesso, di razza, di lingua, di religione, di opinioni politiche, di condizioni personali e sociali. 2. È compito della Repubblica rimuovere gli ostacoli di ordine economico e sociale, che, limitando di fatto la libertà e l'eguaglianza dei cittadini, impediscono il pieno sviluppo della persona umana e l'effettiva partecipazione di tutti i lavoratori all'organizzazione politica, economica e sociale del Paese".

${ }^{19} \mathrm{Cfr}$. il § 3. II., lett. b) dell'Ordinanza di rimessione, p. 66. Secondo il Tribunale di Venezia, la disparità di trattamento causata dalle norme in discorso è evidente "laddove privilegia chi dispone dei mezzi economici non solo per concepire, ma anche per far nascere il figlio all'estero e richiedere, con ormai sicuro successo, la trascrizione in Italia dell'atto di nascita straniero".

${ }^{20}$ Il primo comma dell'Articolo 30 prevede che "(è) dovere e diritto dei genitori mantenere, istruire ed educare i figli, anche se nati fuori del matrimonio".

${ }^{21} \mathrm{Si}$ veda, sul punto, il § 3. II., lett. c) dell'Ordinanza di rimessione, p. 66.

${ }^{22}$ Ai sensi del primo comma dell'Articolo 117, “(1)a potestà legislativa è esercitata dallo Stato e dalle Regioni nel rispetto della Costituzione, nonché dei vincoli derivanti dall'ordinamento comunitario e dagli obblighi internazionali". La disposizione rappresenta la clausola generalizzata di rispetto degli obblighi internazionali, ed è stata introdotta nell'ordinamento italiano con la legge di revisione costituzionale n. 3 del 2001.

${ }^{23}$ Cfr. Ordinanza di rimessione, § 3. III., p. 66. 
15. Per il Tribunale di Venezia, è un "principio internazionale definitivamente acquisito quello secondo cui il matrimonio non rappresenta il discrimen" nei rapporti tra genitori - i quali hanno diritto a veder soddisfatto il proprio desiderio di genitorialità anche oltre il limite imposto dalla natura - e i figli, i quali hanno diritto al medesimo grado di tutela, a prescindere dalla forma del legame sussistente tra coloro che ne hanno assunto la genitorialità.

Il combinato disposto tra il comma 20, dell'Articolo 1 della legge 76/2016 e il comma 2 dell'Articolo 29 del D.P.R. n. 396/2000 pregiudicherebbe - secondo il Tribunale rimettente - alcuni diritti inviolabili della persona. In particolare, "il diritto alla genitorialità e il diritto alla procreazione nell'ambito di una unione civile legalmente riconosciuta nell'ordinamento interno" sarebbero compromessi; i cittadini sarebbero discriminati "in ragione del loro orientamento sessuale ed in considerazione delle condizioni patrimoniali in cui versano le coppie"; e verrebbe introdotto "un irragionevole divieto basato su discriminazioni per ragioni legate all'orientamento sessuale della coppia"24.

16. In assenza - come anticipato - di un quadro normativo organico in materia, la piena comprensione della decisione della Corte costituzionale presuppone una ricognizione delle più recenti pronunce giurisprudenziali, tanto di legittimità quanto costituzionali, in materia di procreazione medicalmente assistita e riconoscimento degli status che ne derivano. Solo, difatti, la sintetica delineazione dei vari tasselli giurisprudenziali sin qui resi dalle giurisdizioni superiori consentirà di ricomporre l'elaborato mosaico in materia.

\section{L'evoluzione della giurisprudenza della Corte di cassazione e della Corte costituzionale in materia di omogenitorialità}

17. Il primo tassello è rappresentato dalla sentenza della Corte di Cassazione, n. 12962 del $2016^{25}$, riguardante un caso di stepchild adoption, ovverosia di adozione da parte di una donna del figlio minore della partner omosessuale - madre biologica del nato - con essa stabilmente convivente e con cui aveva condiviso il progetto genitoriale.

Con tale pronuncia, la Corte ha legittimato il ricorso all'adozione in casi particolari, ai sensi dell'Articolo 44, comma 1, lett. d), della legge 184/1983 ${ }^{26}$, per le coppie omosessuali, ovverosia anche in caso di impossibilità giuridica di affidamento preadottivo, stante l'esistenza di un genitore biologico che si prende cura del minore e la conseguente impossibilità di dichiarare quest'ultimo in stato di abbandono.

L'istituto, pertanto, secondo la Cassazione, può trovare applicazione anche nel caso in cui sussista l'interesse concreto del minore a vedersi riconosciuto il rapporto genitoriale instauratosi con il genitore sociale, pur avente lo stesso sesso del genitore biologico.

18. Con le sentenze n. 19599 del $2016^{27}$ e n. 14878 del $2017^{28}$, la Corte di Cassazione è invece pervenuta - tramite la valorizzazione del principio del superiore interesse del minore - ad assicurare la

\footnotetext{
${ }^{24} \mathrm{Si}$ veda il $\S 4$. dell'Ordinanza di rimessione, p. 68.

${ }^{25} \mathrm{Su}$ tale pronuncia, si veda L. Vizzon, "La Cassazione conferma la stepchild adoption della minore da parte della partner omosessuale della madre biologica", in Familia, 2016, disponibile su www.rivistafamilia.it.

${ }^{26}$ Legge 4 maggio 1983, n. 184, in G.U. 17 maggio 1983, n. 133. Ci si soffermerà sui tratti essenziali dell'istituto dell'adozione in casi particolari, nonché sui limiti del suo impiego nei casi di omogenitorialità, infra, parr. 52 ss.

${ }^{27}$ Corte di cassazione, prima sezione, sentenza 21 giugno 2016, n. 19599/2016, depositata il 30 settembre 2016. Sulla pronuncia, si veda O. FERACI, "Ordine pubblico e riconoscimento in Italia dello status di figlio "nato da due madri” all'estero: considerazioni critiche sulla sentenza della Corte di cassazione n. 1599/2016", in Rivista di diritto internazionale, 2017, pp. 169-181. L'Autrice rileva criticamente che la sentenza rappresenta "un revirement giurisprudenziale rispetto all'impostazione classica sulla natura e sul funzionamento del limite internazionalprivatistico dell'ordine pubblico", nel senso di una sua costituzionalizzazione. In favore, invece, di una visione costituzionalmente orientata dell'ordine pubblico, si veda F. SALERNO, "La costituzionalizzazione dell'ordine pubblico internazionale", in Rivista di diritto internazionale privato e processuale, 2018, pp. 29-61.

${ }^{28}$ Corte di cassazione, prima sezione, sentenza 26 ottobre 2016, n. 14878, depositata il 15 giugno 2017, alla luce della quale l'ordine pubblico in tema di atti di stato civile "attiene ad esigenze di tutela dei diritti fondamentali dell'uomo"; di conseguenza, "la trascrizione in Italia di un atto di stato civile validamente formato all'estero, nel quale risulti la nascita del figlio da due madri non contrasta con l'ordine pubblico (art. 18 del d.P.R. n. 396/2000) (nell' accezione anzidetta) per il fatto che il legislatore nazionale non preveda o vieti tale fattispecie". Si veda, sul punto, F. SALERNo, "La costituzionalizzazione", cit., p. 50.
} 
tutela della continuità transnazionale dello status di figlio legittimamente acquisito all'estero, garantendo la trascrivibilità in Italia, in quanto non contrastante con l'ordine pubblico internazionale, di atti di nascita validamente formati all'estero sulla base del diritto locale, contenenti l'indicazione di ambedue i componenti di una coppia omosessuale femminile.

19. La Corte costituzionale, con la sentenza n. 272 del $2017^{29}$, si è invece occupata di maternità surrogata, in particolare ribadendo il divieto - sanzionato penalmente - di ricorso a tale tecnica di procreazione medicalmente assistita, in quanto potenzialmente lesiva della dignità della donna e della tenuta delle relazioni umane.

In questa prospettiva, la Corte ha legittimato la menzione nell'atto di nascita del nome della madre surrogata, anziché di quello del genitore intenzionale, nell'ottica dell'interesse del minore nato attraverso il ricorso a tale pratica a conoscere la propria identità.

20. La compatibilità della maternità surrogata con il limite dell'ordine pubblico è stata oggetto anche della sentenza della Corte di cassazione, n. 12193 del 2019 ${ }^{30}$, con cui le Sezioni Unite hanno negato il riconoscimento dello status filiationis legittimamente acquisito all'estero a seguito del ricorso a tale tecnica procreativa medicalmente assistita.

Secondo la Corte, il divieto di maternità surrogata costituisce un principio di ordine pubblico, posto a presidio tanto della tutela della dignità umana, quanto dell'istituto dell'adozione. In questa prospettiva, la Corte ha ribadito che il rapporto genitoriale di intenzione può trovare riconoscimento attraverso l'istituto dell'adozione in casi particolari, disciplinato dall'Articolo 44, comma 1, lett. d) della legge $184 / 1983^{31}$.

21. Sempre nel 2019, la Corte costituzionale, nella sentenza n. $221^{32}$, ha respinto le censure di illegittimità costituzionale rivolte ad alcuni Articoli ${ }^{33}$ della legge 40/2004, indicando che l'esclusione dalla procreazione medicalmente assistita delle coppie formate da due donne non dà vita ad una ingiustificata disparità di trattamento tra coppie omosessuali ed eterosessuali.

In particolare, la Corte ha sancito l'inesistenza di un "diritto a procreare", tenendo in considerazione, per un verso, la funzione delle tecniche procreative medicalmente assistite - ovverosia quella di porre rimedio a forme di infertilità o sterilità patologiche - e, per altro verso, "la struttura del nucleo familiare delle tecniche in questione" - ossia quello caratterizzato dalla presenza di un padre ed una madre.

\footnotetext{
${ }^{29}$ Corte costituzionale, sentenza 22 novembre 2017 n. 272, depositata il 18 dicembre 2017, in G.U. 20 dicembre 2017, n. 51, disponibile su www.cortecostituzionale.it. Sulla sentenza, si veda, G. MATUCCI, "La dissoluzione del paradigma della verità della filiazione innanzi all'interesse concreto del minore (Nota a sent. Corte cost., 18 dicembre 2017, n. 272)", in Forum di Quaderni Costituzionali, vol. 2, 2018; G. BARCELlona, "La Corte e il peccato originale: quando le colpe dei padri ricadono sui figli. Brevi note a margine di Corte cost. 272 del 2017", in Forum di Quaderni Costituzionali, vol. 3, 2018. Ambedue i contributi sono disponibili su www.forumcostituzionale.it/.

${ }^{30}$ Sulla sentenza, si vedano F. MarongiU Buonaiuti, "Recognition in Italy of filiation established abroad by surrogate motherhood, between transnational continuity of personal status and public policy", in Cuadernos de Derecho Transnacional, 2019, vol. 2, pp. 294-305; O. FeraCI, "La nozione di ordine pubblico alla luce della sentenza della Corte de cassazione (sez. un. civ.), n. 12193/2019 tra 'costituzionalizzazione attenuata' e bilanciamento con il principio del superiore interesse del minore" in Rivista di diritto internazionale, 2019, pp. 1137-1151. Secondo l'Autrice, le Sezioni Unite hanno fornito fornendo una rilettura del limite dell'ordine pubblico, nell'ottica di una costituzionalizzazione attenuata e della complementarità delle fonti internazionali e sovranazionali ai fini della ricostruzione dello stesso.

${ }^{31}$ Si veda, infra, parr. 52 ss.

${ }^{32}$ Corte costituzionale, sentenza 19 giugno 2019 n. 221, depositata il 23 ottobre, in G.U. 30 ottobre 2019, n. 44, disponibile su www.cortecostituzionale.it. Sulla pronuncia, si vedano, ex multis, M. PICCHI, "Il divieto per le coppie omosessuali di accedere alla PMA: la Corte costituzionale compie un'interpretazione autentica della pregressa giurisprudenza. (Riflessioni sulla sentenza n. 221/2019)", in Forum di Quaderni Costituzionali, vol. 2, 2020, pp. 143-165, disponibile su www.forumcostituzionale.it; U. SAlANITRo, "A strange loop. La procreazione assistita nel canone della Corte costituzionale", ne Le nuove leggi civili commentate, vol. 1, 2020, pp. 206-219; M. TRAPANI, "Il divieto di inseminazione artificiale di tipo eterologo per le coppie omogenitoriali tra scienza e coscienza sociale", in dirittifondamentali.it, fasc. 2, 2020, pp. 804-827, disponibile su www.dirittifondamentali.it.

${ }^{33}$ Le censure erano rivolte, in particolare, agli Articoli 5, 12, commi 2, 9 e 10, nonché all'Articolo 1, commi 1 e 2, e 4 della legge 40/2004, per asserito contrasto con gli Articoli 2, 3, 11, 31, comma 2, 31, comma 1 e 117, comma 1, della Costituzione, quest'ultimo in relazione agli articoli 8 e 14 della Convenzione europea dei diritti dell'uomo.
} 
In questa prospettiva, la Corte costituzionale ha inoltre rammentato la pronuncia della Corte $\mathrm{EDU}^{34}$ secondo cui non può essere ritenuta causa di una ingiustificata disparità di trattamento, rilevante ai fini degli Articoli 8 e 14 della Convenzione, una legge nazionale che riservi l'accesso alle tecniche di procreazione medicalmente assistita esclusivamente alle coppie eterosessuali sterili, attribuendole dunque una finalità terapeutica, non essendo la situazione di infertilità fisiologica della coppia omosessuale comparabile con quella patologica delle coppie eterosessuali.

22. Nella sentenza n. 237 del $2019^{35}$, la Corte costituzionale si è pronunciata in relazione alla vicenda di una madre gestazionale cittadina statunitense e una madre intenzionale cittadina italiana. Quest'ultima aveva espresso il proprio consenso alla fecondazione eterologa avvenuta in Danimarca con seme di un donatore anonimo. Il Tribunale rimettente aveva sollevato una questione di legittimità in relazione all'impossibilità di formare in Italia un atto di nascita in cui siano riconosciute come genitori di un minore straniero due persone dello stesso sesso, quando la filiazione sia stabilita sulla base della legge straniera applicabile. Secondo il giudice rimettente, la legge del Winsconsin - richiamata ai sensi dell'Articolo 33 della legge 218/1995 36 in quanto legge nazionale del figlio, cittadino statunitense - considera genitore anche chi ha dato l'assenso alla fecondazione eterologa.

La Corte ha dichiarato il ricorso inammissibile, a causa della non precisa individuazione dell'oggetto della domanda, ovverosia del contenuto della norma di applicazione necessaria della cui compatibilità con i precetti costituzionali si faceva questione.

Sotto un diverso profilo, nella sentenza in parola, riprendendo quanto già chiarito nella sentenza 221 del 2019 in relazione alla legge n. 40/2004 in materia di procreazione medicalmente assistita, la Corte ha inoltre precisato che la legge n. 76/2016 in materia di unioni civili non consente di pervenire a conclusioni diverse: pur riconoscendo dignità giuridica e sociale alle coppie costituite da individui dello stesso sesso, non consente la filiazione, né adottiva né per fecondazione assistita, in loro favore ${ }^{37}$.

23. Da ultimo, con la sentenza del 3 aprile 2020 , n. $7668^{38}$, la Corte di cassazione ha stabilito che non può trovare accoglimento la domanda di rettificazione dell'atto di nascita di un minore nato in Italia, attraverso l'inserimento del nome della madre intenzionale accanto a quello della madre biologica, sebbene la prima avesse in precedenza acconsentito alla procreazione medicalmente assistita eseguita all'estero, poiché per le persone dello stesso sesso vige, nell'ordinamento italiano, il divieto di ricorso a tale tecnica riproduttiva.

\section{L'orientamento della Corte costituzionale nella sentenza n. 230 del 2020}

24. La ricostruzione giurisprudenziale sin qui delineata consente, ora, di meglio comprendere le ragioni sottese alla pronuncia di inammissibilità della questione di legittimità rimessa alla Corte costituzionale da parte del Tribunale di Venezia.

\footnotetext{
${ }^{34}$ Corte europea dei diritti dell'uomo, sentenza 15 marzo 2013, Gas e Dubois c. Francia, ricorso n. 25951/07, parr. 68 ss.

${ }^{35}$ Corte costituzionale, sentenza 21 ottobre 2019 n. 237, depositata il 15 novembre 2019, in G.U. 20 novembre 2019, n. 47, disponibile su www.cortecostituzionale.it. A commento della sentenza si veda A. CANNONE, "Norme italiane in tema di fecondazione eterologa per coppie same-sex: norme di applicazione necessaria?", in Rivista di diritto internazionale, vol. 2 , 2020, pp. 563-568.

${ }^{36}$ Secondo l'Articolo 33 della legge 218/1995 di riforma del sistema di diritto internazionale privato, "1. Lo stato di figlio è determinato dalla legge nazionale del figlio o, se più favorevole, dalla legge dello Stato di cui uno dei genitori è cittadino, al momento della nascita".

${ }^{37} \mathrm{Si}$ veda, in particolare, il par. 3.1.1. della sentenza n. 237 della Corte costituzionale.

${ }^{38}$ Corte di cassazione, prima sezione, sentenza 3 aprile 2020 n. 7668. Sulla sentenza si veda R. TREzZA, "P.M.A. all'estero e nascita in Italia: ha diritto di essere menzionata nell'atto di nascita soltanto la madre biologica e non anche quella intenzionale", in Familia, 2020, disponibile sul sito www.rivistafamilia.it. Sia inoltre permesso il richiamo al post "Sulla rettificazione dell'atto di nascita attraverso l'indicazione del nome della madre intenzionale a seguito di procreazione medicalmente assistita", pubblicato su AldricusBlog, www.aldricus.giustizia.it
} 
25. In particolare, la Corte ha ritenuto corretta premessa esegetica dalla quale muoveva il giudice a quo. Non è difatti possibile, secondo la Corte, superare in via interpretativa l'ostacolo - rappresentato dal dato testuale dell'Articolo 5 della legge 40/2004 - che consente l'accesso alle tecniche di procreazione medicalmente assistita esclusivamente alle coppie di sesso diverso, "atteso che le coppie dello stesso sesso non possono accedere, in Italia, alle tecniche di procreazione medicalmente assistita".

26. Dopo aver rammentato le precedenti sentenze n. $221^{39}$ e n. 237 del $2019^{40}$, nonché la sentenza n. 7668/2020 della Corte di cassazione ${ }^{41}$, la Corte costituzionale ha sancito che gli Articoli 2, 3 e 30 della Costituzione, nonché i parametri convenzionali ed europei richiamati per il tramite dell'Articolo 117, primo e secondo comma, non autorizzano "l'auspicato 'riconoscimento delle donne omosessuali civilmente unite quali genitori del nato da fecondazione eterologa praticata dall'una con il consenso dell'altra"'42.

27. Secondo la Corte, inoltre, nonostante l'ampio dibattito che ha preceduto l'adozione della legge 76/2016, il legislatore italiano ha comunque scelto di non riferire le norme sulla filiazione delle coppie omosessuali, di fatto sottendendo la "non [...] arbitraria o irrazionale idea, già richiamata nella sentenza n 221 del 2019, secondo cui la "famiglia ad instar naturae - due genitori di sesso diverso, entrambi viventi e in età potenzialmente fertile - rappresenta, in linea di principio, il 'luogo' più idoneo ad accogliere e crescere il nuovo nato", non violando gli Articoli 2 e 30 della Costituzione ${ }^{43}$.

28. La Corte ha altresì ribadito l'inesistenza di un diritto fondamentale alla genitorialità. In particolare, secondo la Consulta, la libertà della filiazione, garantita dall'Articolo 30 della Costituzione, deve essere nondimeno bilanciata "con altri interessi costituzionalmente protetti" ${ }^{4}$.

29. Quanto alla ipotizzata violazione dell'Articolo 3, ancorché la giurisprudenza, anche di legittimità, ammetta "il riconoscimento in Italia di atti formati all' estero, dichiarativi del rapporto di filiazione nei confronti di 'due madri'", secondo la Corte l'ordinamento italiano non può tenere in considerazione il fatto che esista una differenza tra la normativa italiana e le normative mondiali, poiché altrimenti la disciplina nazionale dovrebbe essere sempre allineata alla più permissiva tra le normative estere in materia ${ }^{45}$.

30. La Corte ha altresì rammentato che, in materia di famiglia, tanto la Convenzione europea dei diritti dell'uomo quanto la Carta di Nizza, fanno esplicito rinvio alle discipline nazionali, lasciando agli Stati un ampio margine di apprezzamento per le questioni etiche e morali ${ }^{46}$. La Corte ha inoltre ripreso il parere consultivo emesso dalla Corte europea dei diritti dell'uomo ${ }^{47}$, in materia di riconoscimento del legame tra genitore d'intenzione e figlio. Secondo la Corte europea, al fine di stabilire il legame tra la madre intenzionale e il figlio nato da maternità surrogata, gli Stati non sono tenuti a registrare nel certificato di nascita il nome della donna, ben potendo l'adozione costituire il mezzo per assicurare il

\footnotetext{
${ }^{39}$ V. supra, par. 21.

${ }^{40}$ Cfr. supra, par. 22.

${ }^{41}$ Si veda, supra, par. 23.

${ }^{42} \mathrm{Si}$ veda, Corte costituzionale, sentenza n. 230 del 2020, cit., par. 6.

${ }^{43}$ Cfr. Corte costituzionale, sentenza n. 230 del 2020, cit., par. 6.

${ }^{44}$ Corte costituzionale, sentenza n. 230 del 2020, cit., par. 6.

${ }^{45}$ Corte costituzionale, sentenza n. 230 del 2020, cit., par. 6. La Corte si era già espressa in questo senso della sentenza 221 del 2019. Si veda, supra, par. 21.

${ }^{46}$ Corte europea dei diritti dell'uomo, seconda sezione, sentenza 28 agosto 2012, Costa e Pavan c. Italia, ricorso $\mathrm{n}$. 54270/10; Grande camera, sentenza 3 novembre 2011, S.H. c. Austria, ricorso n. 57813/00.

${ }^{47}$ Corte europea dei diritti dell'uomo, Parere consultivo del 10 aprile 2019, relativo al riconoscimento nel diritto interno del legame di filiazione tra un figlio nato da una gestazione per altri praticata all'estero e la madre intenzionale, sulla base del Protocollo n. 16 annesso alla CEDU su richiesta della Cour de Cassation (domanda n. P16-2018-001), disponibile all'indirizzo www.hudoc.echr.coe.int. Sul parere, si veda O. FeracI, "Il primo parere consultivo della CEDU su richiesta di un Giudice nazionale e l'ordinamento giuridico italiano", in Osservatorio sulle fonti, vol. 2, 2019, disponibile su www.osservatoriosullefonti. it. Si veda anche A. Di BLASE, "Il riconoscimento della genitorialità a favore del genitore non biologico nel parere della Corte europea dei diritti dell'uomo del 10 aprile 2019, in SidiBlog, reperibile su www.sidiblog.org/.
} 
riconoscimento di tale legame, purché rispetti i canoni di effettività e celerità ${ }^{48}$, nel rispetto del superiore interesse del minore.

31. La Corte ha tuttavia precisato che i precetti costituzionali non sono chiusi a soluzioni di segno diverso ${ }^{49}$; spetta tuttavia al legislatore - e non alla Corte costituzionale - l'adozione di una normativa tesa a consentire il riconoscimento dell'omogenitorialità. Tale svolta, lungi dall'essere costituzionalmente imposta, rientra, secondo la Corte, nella discrezionalità del legislatore in qualità di "interprete della volontà della collettività" 50 .

32. Da ultimo, in riferimento al vulnus asseritamente arrecato all'interesse del minore, la Corte ha ribadito che tale interesse è stato preso in considerazione dalla giurisprudenza anche di legittimità, in particolare attraverso l'istituto dell'adozione non legittimante, ai sensi dell'Articolo 44, comma 1, lett. d) della legge 184/1983. Seppur possibile, una diversa, maggiore tutela del superiore interesse del minore ricade comunque, secondo la Corte, nella sfera di discrezionalità del legislatore.

\section{Considerazioni critiche}

33. La pronuncia della Corte costituzionale - che, invero, riecheggia la sentenza n. 7668/2020 resa dalla Corte di cassazione ${ }^{51}$ - è foriera di alcune considerazioni critiche ${ }^{52}$, latamente riconducibili a quattro profili.

In primo luogo, appare censurabile la decisione della Corte costituzionale di ricorrere ad una decisione di inammissibilità. In secondo luogo, permane il vulnus arrecato alla tutela del superiore interesse del minore, atteso che l'ampiezza del grado di tutela lui accordato dipende da fattori essenzialmente secondari rispetto all'esigenza primaria di tutela del suo superiore interesse. In terzo luogo, occorre mettere in luce la portata variabile che l'ordine pubblico internazionale in materia viene ad assumere. In quarto luogo, appare censurabile il perdurante ricorso all'istituto dell'adozione in casi particolare ai fini della regolamentazione del rapporto tra genitore intenzionale e figlio.

\footnotetext{
${ }^{48} \mathrm{Si}$ veda, in particolare, il paragrafo 55 del parere consultivo, nel quale la Corte europea dei diritti dell'uomo ha precisato che "given the margin of appreciation available to States as regards the choice of means, alternatives to registration, notably adoption by the intended mother, may be acceptable in so far as the procedure laid down by domestic law ensures that they can be implemented promptly and effectively, in accordance with the child's best interests".

${ }^{49}$ Secondo la Corte rileva, in questo senso, anche l'oramai riconosciuta non contrarietà all'ordine pubblico della trascrizione di certificati di nascita formati all'estero, con annotazione della duplice genitorialità femminile.

${ }^{50} \mathrm{Si}$ veda, Corte costituzionale, sentenza n. 230 del 2020, cit., par. 8.

${ }^{51}$ Cfr. supra, par. 23.

${ }^{52}$ Sulla sentenza n. 230 del 2020 si vedano, inter alia, G. CiCCHETTI, "L'omogenitorialità 'fra realtà fattuale e realtà legale' (nota a C. Cost., sent. 230/2020)", in iusinitinere.it; S. STEFANELLI, "Il rapporto tra nato in Italia da p.m.a. e madre intenzionale resta confinato all'adozione genitoriale", in Il Foro Italiano, fasc. 1, 2021, pp. 44-68; C. Bona, "La filiazione omosessuale, tra $<<$ rivoluzione arcobaleno $>>$ e diritto $<<$ postmoderno $>>$, in Il foro italiano, fasc. 1, 2021, pp. 39-43; M.R. DonNARUMMA, "Le "questions de societé": confine tra sindacato di costituzionalità e discrezionalità del legislatore", in Diritto penale e uomo, vol. 1, 2021, disponibile su www.dirittopenaleuomo.org/; E. Olıviтo, "(Omo)genitorialità intenzionale e procreazione medicalmente assistita nella sentenza n. 230 del 2020: la neutralità delle liti strategiche non paga", in Osservatorio costituzionale, fasc. 2 , 2021, disponibile a questo indirizzo: www.osservatorioaic.it/; M. PICCHI, "Figli di un dio minore': quando lo status filiationis dipende dal luogo di nascita (Brevi riflessioni a margine della sentenza n. 230/2020 della Corte costituzionale)", in Forum di Quaderni Costituzionali, 2021, disponibile su www.forumcostituzionale.it/; C. TRAPUZZANO, "Unioni civili: il riconoscimento della filiazione verso due madri spetta al legislatore", ne Il Quotidiano Giuridico, 2021, disponibile su www.quotidianogiuridico.it; V. A. Di Martino, "Corte costituzionale, atti di nascita di bambini nati nell'ambito di una coppia lesbica e accesso alla P.M.A.”, in Diritti comparati, 2020, reperibile al sito www.diritticomparati.it; S. Spatola, "Il giudice delle leggi dice 'no' al riconoscimento dell'omogenitorialità per via estensiva: non è la Corte costituzionale il luogo ma il Parlamento. A margine della sentenza n. 230 del 2020 della Consulta", in Federalismi.it, fasc. 35, 2020, pp. 105-117, reperibile su www.federalismi.it.; A. GIUBILEI, "L'aspirazione alla genitorialità delle coppie omosessuali femminili. Nota alla sentenza n. 230 del 2020 della Corte costituzionale", in Nomos - Le attualità nel diritto, vol. 3, 2020, reperibile su www.nomos-leattualitaneldiritto.it/.
} 


\section{L'eccessiva cautela mostrata dalla Corte costituzionale}

34. Anzitutto - ancorché su un piano per lo più attinente al diritto costituzionale - appare criticabile la cautela mostrata dalla Corte costituzionale nell'adozione della decisione in discorso. Se è vero che una risposta omnicomprensiva alle delicate questioni poste dall'intersezione fra le nuove frontiere della genitorialità e gli interrogativi di ordine morale e giuridico che ne conseguono non può essere affidata a decisioni giurisprudenziali giocoforza settoriali, l'approdo raggiunto dalla Consulta sembra essere fin troppo cauto.

35. Come già precisato, la Corte costituzionale non ha escluso soluzioni di segno diverso, pur demandandone l'adozione al legislatore. Non si può tuttavia non tenere in considerazione la riluttanza del legislatore italiano nei confronti degli interventi normativi tesi a colmare lacune giuridiche in relazione a questioni eticamente sensibili, legate all'evoluzione della coscienza sociale ${ }^{53}$. Ne sono esempi, per un verso, la difficoltà nell'adozione di una disciplina dedicata alle unioni civili omosessuali ${ }^{54}$, e la perdurante assenza di una normazione organica relativa al fine-vita ${ }^{55}$.

In questo senso, appare opportuno chiedersi se la Corte costituzionale non avrebbe potuto promuovere un dialogo interistituzionale con il legislatore attraverso l'adozione di una sentenza monitoria $^{56}$, sollecitando un suo intervento al fine di colmare le lacune normative esistenti.

\section{Il vulnus alla tutela del superiore interesse del minore}

36. In secondo luogo, appare opportuno evidenziare il permanere del vulnus alla tutela del superiore interesse del minore, sia da un punto di vista formale che sostanziale.

37. Sin una prospettiva formale, la lesione alla tutela del superiore interesse del minore è essenzialmente legata alla stessa assenza, nell'ordinamento italiano, di una disciplina organica in materia di omogenitorialità.

Nel silenzio del legislatore, l'affastellarsi di decisioni - rese da giudici di merito, di legittimità e costituzionali - rende complessa la ricostruzione della disciplina normativa in tale ambito. L'approccio "a mosaico" cui sono costrette le varie Corti nazionali al fine di colmare il vuoto normativo rappresenta, in quanto tale, un vulnus al principio del superiore interesse del minore, non consentendo una ragionevole prevedibilità in riferimento alle forme in cui si tale tutela possa - e debba - esprimersi.

38. Da un punto di vista sostanziale, la giurisprudenza sin qui elaborata fa sì che l'ampiezza della tutela dello status filiationis finisca per dipendere essenzialmente da due fattori, peraltro del tutto secondari rispetto all'obiettivo - che dovrebbe essere primario - di tutela del superiore interesse del minore nato attraverso procreazione medicalmente assistita.

Tali fattori, presi singolarmente, sono, da un lato, il luogo di nascita del minore ${ }^{57} \mathrm{e}$, dall'altro, la tecnica procreativa medicalmente assistita di volta in volta impiegata. Dall'intersezione di questi due elementi, in particolare, discendono livelli di tutela del rapporto tra genitori intenzionali e figli più o meno ampi.

\footnotetext{
${ }^{53}$ M.R. Donnarumma, "Le 'questions de société"”, cit., p. 2.

${ }^{54}$ Come già anticipato, il legislatore italiano è pervenuto ad una disciplina delle unioni civili omosessuali solo nel 2016, a seguito di un lungo dibattito parlamentare.

${ }^{55} \mathrm{Si}$ veda, sul punto, A. Massaro, Questioni di fine vita e diritto penale, Torino, Giappichelli, 2020.

${ }^{56} \mathrm{Si}$ veda, A. Giubilei, "L'aspirazione alla genitorialità", cit., pp. 13 ss.; M.R. Donnarumma, "Le "questions de societé", cit., p. 15.

${ }^{57}$ M. Picchi, "Figli di un dio minore", cit., p. 7; G. Cicchetti, "L'omogenitorialità", cit.
} 
39. In questa prospettiva, allo stato attuale, la tutela più ampia allo status filiationis è accordata dall'ordinamento italiano all'ipotesi in cui il concepimento del minore sia avvenuto attraverso la tecnica procreativa della fecondazione eterologa, e la nascita sia avvenuta in uno Stato straniero ${ }^{58}$.

Altrimenti detto, un certificato di nascita legittimamente formato all'estero, sulla base del diritto locale, nel quale due donne omosessuali vengano indicate come genitori del minore, potrà circolare oltre le frontiere dello stato di origine e trovare riconoscimento nell'ordinamento italiano, non trovando un limite nell'ordine pubblico del foro. Del resto, la Corte di cassazione è pervenuta a questa soluzione nelle già menzionate sentenze n. 19599/16 e 14878/201759, attraverso il rilievo accordato al superiore interesse del minore, nella prospettiva di assicurare la circolazione dello status legittimamente acquisito all'estero.

Le due pronunce sono menzionate anche dalla Corte costituzionale nella sentenza in commento: nell'evocare l'eventuale apertura dei parametri costituzionali a soluzioni diverse rispetto a quelle sin qui adottate, e nel rammentare che la valutazione sul punto spetta in ultima analisi al legislatore, la Corte ha difatti accordato rilievo al fatto che la trascrivibilità in Italia di certificati di nascita formati all'estero, contenenti una duplice genitorialità femminile, è stata riconosciuta con contraria all'ordine pubblico internazionale, secondo le norme di diritto internazionale privato.

40. Una tutela diversa, senz'altro deteriore, è offerta dall'ordinamento italiano al minore nato all'estero, attraverso il ricorso alla maternità surrogata - sia essa tradizionale o gestazionale, altruistica o commerciale.

In questo secondo caso, come anticipato, la Corte di cassazione ha impedito la trascrivibilità dell'atto di nascita riportante l'indicazione di due padri, alla luce della contrarietà della gestazione per altri con l'ordine pubblico del foro ${ }^{60}$. Secondo l'orientamento oramai consolidato della Corte di cassazione, il rapporto genitoriale di intenzione può trovare valorizzazione tramite l'istituto dell'adozione in casi particolari, disciplinato dall'Articolo 44, comma 1, lett. d) della legge 184/1983. Vieppiù, nella prospettiva della Corte costituzionale ${ }^{61}-\mathrm{e}$, a ben vedere, prescindendo dal legame fattuale tra il minore e i genitori d'intenzione - il certificato di nascita di un minore nato a seguito di gestazione per altri dovrebbe valorizzare il nome della madre surrogata, in vista della tutela dell'interesse del minore a conoscere le proprie origini.

In questa prospettiva, il superiore interesse del minore verrebbe ad essere declinato in funzione non tanto della tutela del legame concreto, fattuale, con i soggetti che di lui si prendono cura, quanto con il diritto del minore in questione a conoscere la donna che ha portato a termine la gestazione.

41. Una tutela ancora diversa è riservata al minore, figlio di una coppia omosessuale, sia esso concepito attraverso la tecnica della fecondazione eterologa ovvero tramite il ricorso ad una surroga di maternità, la cui nascita sia avvenuta in Italia.

Secondo l'approccio delineato dalla Corte costituzionale nella sentenza in commento, e dalla Corte di cassazione nella sentenza $7668 / 20^{62}$, il certificato di nascita formato in Italia non potrà riportare l'indicazione della genitorialità omosessuale. In altre parole, nel caso in cui una coppia omogenitoriale femminile abbia fatto ricorso alla fecondazione eterologa all'estero, e la nascita del minore sia avvenuta in Italia, l'atto di nascita potrà contenere l'indicazione della sola madre gestante, ritenuta madre biologica. Anche in questo caso, il rapporto tra genitore intenzionale e figli può essere valorizzato attraverso l'istituto dell'adozione in casi particolari.

\footnotetext{
${ }^{58}$ In riferimento a questa considerazione, nell'ordinanza di rimessione del 3 aprile 2019, n. 108, spec. § 3.2. lett. b), il Tribunale di Venezia fa leva anche su una disparità di trattamento basata sul reddito, legata alle maggiori possibilità di riconoscimento dello status filiationis per quelle coppie che abbiano capacità economiche tali da consentire anche la nascita del minore all'estero. Si veda, supra, nota 19.

${ }^{59}$ Sulle sentenze, si veda supra, par. 18.

${ }^{60}$ Corte di cassazione, sentenza n. 12193 del 2019. Si veda, supra, par. 20.

${ }^{61}$ Corte costituzionale, sentenza n. 272 del 2017. Si veda, supra, par. 19.

${ }^{62}$ Cfr., supra, par. 23.
} 
42. A ben vedere, se l'intento della giurisprudenza - nel silenzio del legislatore - è quello di accordare rilievo al legame biologico anziché a quello cd. "sociale" o "di intenzione", tale strada non appare scevra di rischi. Non sempre, difatti, la madre gestante è anche la madre biologica del minore: nel caso di fecondazione eterologa gestazionale - ovverosia attraverso l'impianto, nell'utero della madre gestante, di un ovocita della madre non gestante fecondato con seme maschile di un donatore - l'indicazione nel certificato di nascita della madre gestante, ritenuta anche biologica, sarebbe fuorviante ${ }^{63}$.

In tale ipotesi, labile verrebbe ad essere la distinzione tra genitorialità biologica e genitorialità d'intenzione, ritenuta invece determinante sia per le giurisdizioni nazionali che per la Corte europea dei diritti dell'uomo ${ }^{64}$.

43. La disparità di trattamento delle situazioni poc'anzi richiamate, e dunque delle tutele accordate dall'ordinamento nazionale ai minori di volta in volta coinvolti, non appare giustificabile. Una tutela del superiore interesse del minore, variabile in relazione al luogo di nascita e alla tecnica procreativa impiegata, appare scarsamente compatibile con il parametro medesimo.

\section{La portata differenziata del limite dell'ordine pubblico}

44. Peraltro, la sentenza n. 230 del 2020 presuppone - a ben vedere, senza farne menzione - delle valutazioni sulla portata del limite dell'ordine pubblico ${ }^{65}$.

45. L'ordine pubblico del foro, nella prospettiva dei giudici nazionali, sia di legittimità che costituzionali, sembra avere una connotazione diversa allorché si tratti di dar vita ad una relazione ritenuta contrastante con principi ritenuti essenziali, ovvero di riconoscere gli effetti della medesima situazione, legittimamente disciplinata sulla base di una legge straniera, e dei diritti e degli status acquisiti all'estero per il tramite della stessa.

46. La portata dell'ordine pubblico internazionale sembra dunque espandersi e restringersi a seconda del luogo in cui la situazione asseritamente contrastante con i valori essenziali del foro viene ad essere costituita.

47. In particolare, l'ordine pubblico del foro ha una valenza piena allorché si tratti di costituire in Italia una situazione contrastante con valori ritenuti fondamentali per l'ordinamento: considerazioni di ordine pubblico, difatti, impediscono la formazione in Italia del certificato di nascita indicante l'omogenitorialità. L'ordine pubblico assume invece una valenza attenuata, invece, allorché si tratti di trascrivere in Italia un certificato di nascita regolarmente formato all'estero, secondo le norme dello Stato di origine.

48. La ricostruzione del limite dell'ordine pubblico in materia di omogenitorialità approntata dalle Corti nazionali, in assenza di una disciplina legislativa in materia, sembra dunque riecheggiare la dottrina francese dell'ordre public atténuét ${ }^{6}$.

49. Allo stesso modo, il limite dell'ordine pubblico torna ad avere portata piena nelle ipotesi di riconoscimento di una situazione legittimamente creata all'estero, nei quali le leggi straniere applicabili abbiano determinato un risultato comunque incompatibile con l'ordine pubblico del foro. È questo il caso, nella materia che ci occupa, della non trascrivibilità in Italia dell'atto di nascita recante

\footnotetext{
${ }^{63}$ Cfr. M. PICCHI, "Figli di un dio minire", cit., spec. p. 6.

${ }^{64} \mathrm{Si}$ veda, al riguardo, Corte europea dei diritti dell'uomo, Parere consultivo del 10 aprile 2019, cit., passim.

${ }^{65}$ R. BARATTA, "Diritti fondamentali e riconoscimento dello "status filii" in casi di maternita surrogata: la primazia degli interessi del minore", in Diritti umani e diritto internazionale, fasc. 2, 2016, p. 309-334, p. 313.

${ }^{66} \mathrm{Si}$ veda, sul punto, O. FeraCI, L'ordine pubblico nel diritto dell'Unione europea, Giuffré, 2012, p. 17 e nota 26.
} 
l'indicazione, in qualità di genitori, di una coppia omosessuale di sesso maschile, in cui pertanto la nascita del figlio sia avvenuta per il tramite di maternità surrogata e nell'impossibilità, a fortiori, di formare un simile certificato di nascita in Italia.

\section{I limiti dell'istituto dell'adozione in casi particolari}

50. Un ulteriore profilo, più volte menzionato nel corso della trattazione, merita ora di essere approfondito.

51. La Corte europea dei diritti dell'uomo si è sovente espressa nel senso della necessità, per gli Stati parte della Convenzione, di riconoscere e garantire il legame tra il genitore biologico e il figlio nato da maternità surrogata ${ }^{67}$. A fortiori, tale legame dovrebbe trovare adeguato riconoscimento e tutela nel caso in cui il minore possa vantare un nesso biologico con la madre non gestante, ovverosia allorché l'ovocita di quest'ultima sia stato impiantato nell'utero della madre gestante.

52. Nel Parere consultivo del 2 aprile 2019, la Grande Camera della Corte europea dei diritti dell'uomo si è occupata, su sollecitazione della Cour de cassation, del riconoscimento di un legame genitoriale tra il figlio nato da maternità surrogata e la madre intenzionale. In particolare, la Grande Camera ha precisato che il margine di apprezzamento lasciato agli Stati non impone loro di indicare il nome della madre intenzionale sul certificato di nascita. In questo senso, gli Stati parti della Convenzione possono avvalersi di alternative alla registrazione, purché la procedura predisposta a livello nazionale assicuri che tali soluzioni possano essere adottate "promptly and effectively, in accordance with the child's best interests".

53. Nel quadro frastagliato dell'ordinamento italiano, la giurisprudenza - di merito, di legittimità e costituzionale - ha più volte indicato nell' istituto dell'adozione in casi particolari ai sensi dell' Articolo 44, comma 1, lett. d) della 1. 184/193 lo strumento adatto a garantire il legame tra genitore intenzionale e figlio nato a seguito di procreazione medicalmente assistita e, dunque a sopperire all'impossibilità, per la madre non gestante, o comunque per il genitore di intenzione, di essere indicata nell'atto di nascita formato all'estero.

54. L'adozione in casi particolari, disciplinata agli Articoli 44 e ss. del codice civile, rappresenta lo strumento attraverso cui può comunque avere luogo il riconoscimento del legame genitoriale, nonostante il minore in questione non sia in stato di abbandono, o comunque l'adozione piena non possa realizzarsi. In particolare, ai sensi della lett. d) del primo comma dell'Articolo 74 può disporsi l'adozione nei confronti del minore "per il quale risulti impossibile l'affidamento preadottivo". L'impossibilità è stata intesa anche in senso giuridico, e, pertanto, tale norma è stata interpretata estensivamente facendovi rientrare anche i casi di c.d. stepchild adoption, ovverosia dell'adozione da parte del genitore intenzionale del figlio del partner, o comunque concepito con il partner ${ }^{68}$.

55. Tuttavia, l'adozione in casi particolari - altrimenti detta adozione c.d. non legittimante - differisce sensibilmente, quanto agli effetti, rispetto all'adozione c.d. piena.

\footnotetext{
${ }^{67}$ Corte europea dei diritti dell'uomo, quinta sezione, sentenze del, sentenze del 26 giugno 2014, Mennesson e Labassee c. Francia, ricorsi nn. 65192/11 e 65941/11; quinta sezione, sentenza del 21 luglio 2016, Foulon e Bouvet c. Francia, ricorsi nn. 9063/14 e 10410/14, parr. 55 ss. Si veda, ex multis, R. BARATta, "Diritti fondamentali”, cit., p. 317 ss; L. D'Avout, "La «reconnaissance» de la filiation issue d'une gestation pour autrui à l'étranger, après les ârrets Mennesson et Labassee", in Recueil Dalloz, 2014, pp. 1806-1810.

${ }^{68} \mathrm{Si}$ veda, G. Rossolillo, "Riconoscimento di status familiari e adozioni sconosciute all'ordinamento italiano", in Diritti umani e diritto internazionale, fasc. 2, 2016, pp. 335-359, pp. 336 ss.
} 
56. Anzitutto, tramite l' istituto in questione, il minore non acquista lo status di figlio dell'adottante, nonostante gli spettino nei confronti di quest'ultimo tutti i diritti propri di un simile rapporto ${ }^{69}$. Lo status di figlio, invece, viene stabilito esclusivamente per il tramite dell'adozione piena, c.d. legittimante.

Inoltre, per il tramite dell'adozione in casi particolari, il minore non instaura un rapporto di parentela con la famiglia del genitore d'adozione, mentre non cessano i rapporti con la famiglia di origine $^{70}$. Tale circostanza ha rilievo dal punto di vista successorio, nel senso che, per un verso, i parenti dell'adottante non possono succedere al minore adottato e, per altro verso, il minore adottato non può succedere ai parenti dell'adottante.

Ancora, ai sensi dell'Articolo 46 della legge 183/1984, l'adozione in casi particolari è subordinata al necessario consenso del genitore che abbia la responsabilità genitoriale nei confronti del minore, con il risultato - anche in caso di omogenitorialità - di fare ricadere sul minore le conseguenze di una eventuale crisi di coppia ${ }^{71}$.

Peraltro, e da un punto di vista procedurale, l'adozione in casi particolari si caratterizza da cadenze temporali ampie, difficilmente compatibili con l'esigenza di assicurare una tutela rapida ed effettiva al legame tra il minore e l'adottante ${ }^{72}$.

57. La breve analisi dell'istituto dell'adozione in casi particolari sin qui condotta ne rende evidenti i limiti intrinseci.

58. Occorre, pertanto, chiedersi se l'istituto rispetti i requisiti di celerità ed efficacia richiesti dalla Corte europea dei diritti dell'uomo nel Parere consultivo del 2 aprile 2019, e se dunque l'istituto possa rappresentare una alternativa alla formazione del certificato di nascita, attraverso l'indicazione del genitore di intenzione, o comunque nel riconoscimento del certificato di nascita, legittimamente costituito all'estero, indicante la genitorialità omosessuale ${ }^{73}$.

59. A ben vedere, la medesima Corte costituzionale ha espresso dubbi sulla capacità dell'istituto dell'adozione in casi particolari, nel quadro di due pronunce recenti, entrambe di inammissibilità.

60. Nella sentenza n. $32 / 2021^{74}$, la Corte costituzionale si è occupata della questione di legittimità sottopostale dal Tribunale di Padova, relativa ad una madre intenzionale che, a causa di una intervenuta crisi con la partner con cui aveva condiviso un progetto genitoriale, e del mancato assenso della madre biologica all'adozione in casi particolari, non riesce a vedere riconosciuto il proprio legame con il minore.

Richiamando la sentenza n. 230 del 2020, la Corte costituzionale ha rammentato di aver "preannunciato l'urgenza di una 'diversa tutela del miglior interesse del minore, in direzione di più penetranti ed estesi contenuti giuridici del suo rapporto con la "madre intenzionale", che ne attenui il divario tra realtà fattuale e realtà legale', invocando l'intervento del legislatore"75. In particolare, secondo la Corte, le questioni sollevate dai giudici patavini, confermano l'impellenza di un intervento normativo da parte del legislatore, poiché "rivelano in maniera tangibile l'insufficienza del ricorso all'adozione in casi particolari, per come attualmente regolato, tant'è che nello specifico caso è resa impraticabile proprio nelle situazioni più delicate per il benessere del minore, quali sono, indubitabilmente, la crisi della coppia e

\footnotetext{
${ }^{69}$ Spettano, dunque, al minore adottato attraverso il ricorso all'adozione in casi particolari, il diritto al mantenimento, all'educazione e all'istruzione.

${ }^{70}$ È tuttavia controverso l'ambito di applicazione dell'articolo 74 del codice civile, come modificato dalla legge 219/2012, di riforma della filiazione. Si veda, ex multis, M. SESTA, "L'unicità dello stato di filiazione e i nuovi assetti delle relazioni familiari", in Famiglia e diritto, 2013, pp. 231-241, p. 236. Si veda inoltre, recentemente, N. ChIRICALLo, "Maternità surrogata e adozione in casi particolari: il doppio "non liquet' della Consulta", in Familia, fasc. 3, 2021, pp. 421-433.

${ }^{71}$ Si veda, infra, par. 60 .

${ }^{72} \mathrm{Si}$ veda, sul punto, N. ChIRICALLo, "Maternità surrogata", cit., p. 429.

${ }^{73} \mathrm{Si}$ veda, ad esempio, M. PICCHI, "Figli di un dio minire", cit., p. 7

${ }^{74}$ Corte costituzionale, sentenza 20 gennaio 2021 n. 32, depositata il 9 marzo 2021, pubblicata in G.U. 10 marzo 2021, n. 10. Sulla sentenza, si veda, G. Ferrando, "Il diritto dei figli di due mamme o di due papà ad avere due genitori. Un primo commento alle sentenze della Corte Costituzionale n. 32 e 33 del 2021", in giustiziainsieme.it.

${ }^{75}$ Cfr. Corte costituzionale, sentenza n. 32 del 2021, par. 2.4.1.3.
} 
la negazione dell'assenso da parte del genitore biologico/legale" ${ }^{76}$. Nella stessa prospettiva, descrivendo i tratti salienti dell'istituto dell'adozione in casi particolari, la Corte costituzionale ha rilevato che essa produce "effetti limitati, visto che non conferisce al minore lo status di figlio legittimo dell'adottante, non assicura la creazione di un rapporto di parentela tra l'adottato e la famiglia dell'adottante [...] e non interrompe i rapporti con la famiglia d'origine".

61. Nella sentenza n. $33 / 2021^{77}$, la Corte costituzionale si è invece occupata della questione di legittimità, sottopostale dalla Corte di cassazione, relativa alla possibilità di riconoscere nell'ordinamento nazionale un provvedimento straniero, che abbia riconosciuto come genitori del minore tanto il genitore biologico, quanto quello di intenzione. Nel caso di specie, si trattava di due uomini uniti civilmente in Italia, che avevano fatto ricorso alla maternità surrogata di tipo gestazionale all'estero.

Secondo la Corte, nel caso di un minore accudito sin dalla nascita da una coppia che ne ha condiviso il progetto genitoriale, il superiore interesse è "quello di ottenere un riconoscimento anche giuridico dei legami che, nella realtà fattuale, già lo uniscono a entrambi i componenti della coppia, ovviamente senza che ciò abbia implicazioni quanto agli eventuali rapporti giuridici tra il bambino e la madre surrogata"78.

Nella prospettiva della Corte, da un lato tali legami costituiscono parte integrante dell'identità del minore e, dall'altro lato, deve poter essere affermata in capo a coloro che si prendono cura del minore "la titolarità giuridica di quel fascio di doveri funzionali agli interessi del minore che l'ordinamento considera inscindibilmente legati all'esercizio di responsabilità genitoriali", non venendo in rilievo un preteso "diritto alla genitorialità" 79 .

In riferimento all'adozione in casi particolari, la Corte ribadisce che essa "costituisce una forma di tutela degli interessi del minore certo significativa, ma ancora non del tutto adeguata al metro dei principi costituzionali e sovranazionali" posti a presidio del superiore interesse del minore.

Secondo la Corte, il contemperamento tra la legittima finalità di disincentivare il ricorso alla maternità surrogata e la necessità di assicurare il rispetto dei diritti dei minori coinvolti, spetta - ancora una volta - alla discrezionalità del legislatore "nella ormai indifferibile individuazione delle soluzioni in grado di porre rimedio all'attuale situazione di insufficiente tutela degli interessi del minore"

62. Anche alla luce delle due pronunce costituzionali da ultimo menzionate, l'istituto dell'adozione in casi particolari appare scarsamente compatibile con i requisiti di celerità ed efficienza individuati dalla Corte europea dei diritti dell'uomo al fine di adeguatamente tutelare il rapporto tra il minore nato da maternità surrogata e il genitore di intenzione.

63. Appare pertanto verosimile che la Corte europea dei diritti dell'uomo possa presto essere investita di un ricorso teso a saggiare la compatibilità dell'istituto in discorso - e, in generale, la tenuta delle soluzioni adottate dalle Corti nazionali nel silenzio del legislatore - con il paramento della Convenzione.

\section{Conclusioni}

64. La disamina sin qui condotta e i rilievi critici delineati consentono di affermare che, perlomeno allo stato attuale, nell'ordinamento italiano l'omogenitorialità rimane riconducibile all'ambito delle aspirazioni, piuttosto che a quello dei diritti ${ }^{81}$.

\footnotetext{
${ }^{76} \mathrm{Ibid}$.

${ }^{77}$ Corte costituzionale, sentenza 28 gennaio 2021 n. 33, depositata il 9 marzo 2021, pubblicata in G.U. 10 marzo 2021, n. 10. Sulla sentenza, si veda il commento di N. ChiRicallo, "Maternità surrogata", cit.

${ }^{78}$ Corte costituzionale, sentenza n. 33 del 2021, par. 5.4.

${ }^{79}$ Ibid.

${ }^{80}$ Corte costituzionale, sentenza 33 del 2021, cit., par. 5.9.

${ }^{81}$ A. GiubiLei, "L'aspirazione”, cit.,
} 
65. Come visto, tale - insoddisfacente - condizione comporta il proliferare delle forme di c.d. turismo riproduttivo. Nella sua circolazione oltre i confini nazionali, l'individuo tuttavia porta con sé un bagaglio di relazioni personali e status familiari il cui riconoscimento e la cui tutela non possono rimanere disattesi, per il mero attraversamento delle frontiere nazionali ${ }^{82}$. In questo senso, gli sforzi degli Stati dovrebbero tesi a far sì che una relazione familiare, legittimamente costituita in uno Stato, possa efficacemente proiettarsi anche oltreconfine, senza che il mero passaggio da uno Stato ad un altro pregiudichi le garanzie offerte dall'ordinamento d'origine. Occorre dunque evitare che vengano a crearsi relazioni familiari claudicanti ${ }^{83}$, facendo sì che alla circolazione delle persone si accompagni la circolazione dei relativi status.

66. Allo stesso modo, appare oramai imprescindibile l'adozione, da parte degli Stati, di misure atte ad assicurare adeguate forme di tutela nei confronti dei minori nati grazie alle diverse forme di procreazione medicalmente assistita. In questa prospettiva, sembra quantomeno irragionevole che le tutele approntate nel quadro dell'ordinamento italiano varino al variare, per un verso, della tecnica produttiva attraverso cui il minore è stato concepito e, per altro verso, del luogo geografico in cui tale minore è nato.

67. In generale, la risposta a problematiche complesse, suscettibili di sollevare rilevanti interrogativi etici ancor prima che giuridici, non può essere affidata ad un mosaico di interventi giurisprudenziali, giocoforza limitati alla specifica situazione di volta in volta considerata.

68. All'auspicabilità di un intervento omnicomprensivo da parte del legislatore, si affianca la necessità, per gli Stati, di tenere in considerazione i vantaggi della cooperazione internazionale, anche in relazione alle nuove forme di genitorialità e della conseguente esigenza di garantire, per un verso, la circolazione degli status venutisi a creare e, per altro verso, la tutela dei soggetti - non solo minori - a vario titolo coinvolti.

Vanno senz'altro in questa direzione, e debbono pertanto essere sostenute, le istanze promosse nel quadro della Conferenza dell' Aja di diritto internazionale privato ${ }^{84}$ tese alla creazione sia di uno strumento generale di diritto internazionale privato relativo sulla filiazione "legale", che di un protocollo separato relativo alla filiazione legale stabilita a seguito di accordi internazionali di maternità surrogata.

\footnotetext{
${ }^{82}$ Sulla continuità internazionale degli status familiari, si vedano F. SALERno, "The Identity and Continuity of Personal Status in Contemporary Private International Law", in Recueil des cours, t. 395, 2019, p. 9-198; R. BARATTA, "La reconnaissance internationale des situations juridiques personnelles et familiales", in Recueil des cours, t. 348, 2010, p. 253-499; P. LAGARDE (a cura di), La reconnaissance des situations en droit international privé, Paris, Pedone, 2013.

${ }^{83}$ Così viene tradotta l'espressione anglofona limping relationships.

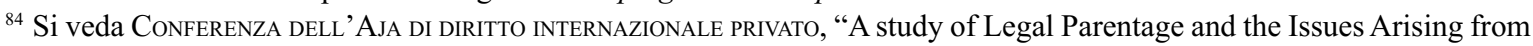
International Surrogacy Arrangements, 2014, disponibile a questo indirizzo < https://assets.hcch.net/docs/bb90cfd2-a66a-4fe4a05b-55f33b009cfc.pdf > . Lo studio è poi stato seguito dall'elaborazione del Progetto Parentage/Surrogacy. Ulteriori informazioni sono reperibili nelle pagine dedicate sul sito della Conferenza dell'Aja: https://www.hcch.net/en/projects/legislative-projects/parentage-surrogacy. A favore di una regolamentazione internazionale dell'istituto, si vedano M. EnGEL, "Cross-Border Surrogacy: Time for a Convention?", in K. Boele-Woelki, N. Dethlof, W. Gephart (a cura di), Family Law and Culture in Europe: Developments, Challenges and Opportunities, Anversa, Intersentia, 2014, pp. 199-216; H. BAKER, “A Possible Future Instrument on International Surrogacy Arrangements: Are There 'Lessons' to be Learnt from the 1993 Hague Intercountry Adoption Convention?”, in K. Trimmings e P. Beaumont (a cura di), International Surrogacy Arrangements: Legal Regulation at the International Level, Oxford, Hart Publishing, 2013, pp. 411-426; E. DAvIs, "The Rise of Gestational Surrogacy and The Pressing Need for International Regulation", in Minnesota Journal of International Law, 2012, pp. 120-141; K. TrImMINGS, P. BEAumont, "International Surrogacy Arrangements: An Urgent Need for Legal Regulation at the International Level", in Journal of Private International Law, vol. 7, 2011, pp. 627-647. Si veda, in proposito, anche lo studio del Parlamento europeo, $A$ Comparative Study on the Regime of Surrogacy in EU Member States, cit., pp. 159 ss.
} 